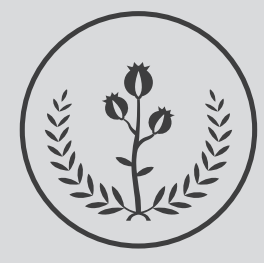

FUCS
Repre

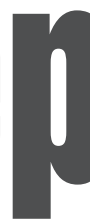

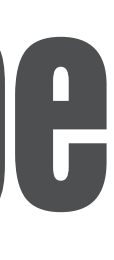

\title{
Simbolismos y enseñanzas médicas y sociales de unas máscaras precolombinas
}

Hugo A. Sotomayor Tribin $\mathrm{MD}^{\mathrm{a}}$

\section{Symbolisms and medical and social teachings of some pre-columbian masks}

${ }^{a}$ Profesor Titular. Fundación Universitaria de Ciencias de la Salud. Bogotá DC, Colombia.

\section{R E S U M EN}

Se presentan 4 máscaras precolombinas, tres de las cuales son ecuatorianas y una colombiana. Las primeras son de cerámica y se adscriben a la cultura Jama-Coaque y la tercera de concha Spondylus prínceps no tiene adscripción a ninguna en particular del Ecuador prehispánico, por no existir un estudio sistemático en Ecuador sobre la relación de esas máscaras con sus culturas costeras, la colombiana es de cobre. La primera de cerámica ecuatoriana por su tamaño, peso y elementos agregados - un material blanco que simula los dientes, tres piedras verdes que parecen los ojos y una incrustación sublabial, un bezote - hacen pensar en una máscara mortuoria de un personaje de poder y alto estatus social; la segunda de cerámica por presentar dieciséis nódulos faciales remite al observador a la llamada verruga peruana o bartonelosis, la tercera ecuatoriana fue elaborada posiblemente como un elemento votivo o de ofrenda para exhibir poder o invocar fuerza o protección dado el carácter de gran valor simbólico de esa concha roja. La máscara de cobre del Cauca medio o Quimbaya, en razón a su material, peso y color, con bastante probabilidad fue usada como adorno colocado sobre el pecho del personaje poderoso o de elevado estatus. Se compara la máscara con bartonelosis con otras dos que el autor ya había documentado, la de concha con otra del mismo tipo de material, la máscara de cobre con una pequeña de piedra jadeíta se referencia con una calavera enmascarada que el autor tuvo la oportunidad de estudiar hace varios años. Se concluye sugiriendo los usos tradicionalmente asignados a las máscaras: festivos, mortuorios y como adornos corporales para resaltar el poder de

INFORMACIÓN DEL ARTÍCULO

Historia del artículo:

Fecha recibido: septiembre 7 de 2020

Fecha aceptado: diciembre 3 de 2020
Autor para correspondencia. Dr. Hugo Sotomayor Tribín husotri@gmail.com
DOI

10.31260/RepertMedCir.01217372.1165 
quien las exhibe. Las máscaras con la erupción tipo nódulos debieron jugar un papel similar a las estatuillas en cerámica y piedra que representan patologías con tanto realismo en el arte prehispánico, pudiendo ser una forma de enseñar, dentro de un pensamiento médico empírico analógico, como lo son los moldes en cera, las fotografías, los dibujos y pinturas en la medicina moderna basada en un pensamiento empírico analítico.

Palabras claves: máscaras prehispánicas, cerámica, concha, piedras, metal poder, rituales, transformación, paleopatología, bartonelosis, enseñanza.

(C) 2021 Fundación Universitaria de Ciencias de la Salud - FUCS. Este es un artículo Open Access bajo la licencia CC BY-NC-ND (http://creativecommons.org/licenses/by-nc-nd/4.0/).

A BSTRACT

Four pre-Columbian masks are presented, three of which are Ecuadorian and one Colombian. The Ecuadorian masks are made of ceramics and ascribe to the Jama-Coaque culture and the third is made of Spondylus prínceps shell, and does not have any ascription to any particular Ecuadorian pre-Hispanic coastal culture, for no systematic study was conducted in Ecuador on the relationship of those masks and coastal cultures. The Colombian mask is made of copper. The first ceramic mask, for its size, weight and additional elements - a white material that simulates teeth, three green stones that simulate eyes and a lower labial incrustation, "bezote"- makes one recall a death mask of an individual with significant power and high social status; the second ceramic mask, features sixteen facial nodules which refer the observer to the Peruvian wart or bartonellosis, the third mask was possibly created as a volitional or offering element to exhibit power or evoke the force or protection given the great symbolic value of that red shell. The middle Cauca Quimbaya copper mask was most probably used as an ornament for the chest of a powerful or high class person given its material, weight and color.

The mask that resembles bartonellosis is compared with other two masks the author had already examined. The shell mask was compared with another made of the same type of shell, the copper mask with a small jadeite stone mask, a masked skull that the author had the opportunity to examine several years ago.

Finally, these interpretations suggest that in addition to the uses traditionally assigned to the masks: festive, funerary and as body adornments to portray the power of the wearer, the masks with the multiple nodular lesions must have served a role similar to that of the ceramic and stone miniature statues which depict pathologies, featuring great realism in pre-Hispanic art, as a way of teaching through an analogical empirical medical thought, the same role played by wax molds, photographs and drawings and paintings in modern medicine, also based on analytical empirical thought.

Keywords: pre-Columbian, Jama-Coaque culture, Spondylus prínceps

(C) 2021 Fundación Universitaria de Ciencias de la Salud - FUCS. This is an open access article under the CC BY-NC-ND license (http://creativecommons.org/licenses/by-nc-nd/4.0/).

\section{IN T ROD U C C IÓN}

Después de publicar en 1994 el artículo “A propósito de la bartonelosis en una máscara ecuatoriana precolombina" en la revista Medicina de la Academia Nacional de Medicina de Colombia ${ }^{1}$ (figura 1), de escribir con la coautoría del arqueólogo Gonzalo Correal Urrego, el artículo "Las calaveras enmascaradas de las momias yuko-yukpa (motilones)" en la Revista de La Academia de Ciencias Exactas, Físicas y Naturales en $2003^{2}$ (figuras 2,3), de haber mostrado en 2012 en mi libro "Aspectos históricos y geográficos de algunas enfermedades importantes en Colombia" en el capítulo "Bartonelosis" una máscara Nariño compatible con la erupción típica de la forma verrugosa y nodular de esa enfermedad $^{3}$ (figura 4), de estudiar un importante número de máscaras precolombinas de diverso material, como lo analizó en su trabajo "Máscara precolombina" el arqueólogo Álvaro Chávez Mendoza en $1974^{4}$ con diferentes expresiones faciales y funciones, y de haber participado en el estudio de la maravillosa colección de máscaras ecuatorianas de material malacológico (figura 5), la mayoría de conchas Spondylus prínceps, de propiedad de Alonso Restrepo de León y que forman parte de su libro inédito titulado " Los Rostros de la $\mathrm{Mar}^{\prime \prime}$, recientemente tuve la suerte de adquirir las cuatro interesantes máscaras motivo de esta breve comunicación (figuras 6,7,8 y 9). 


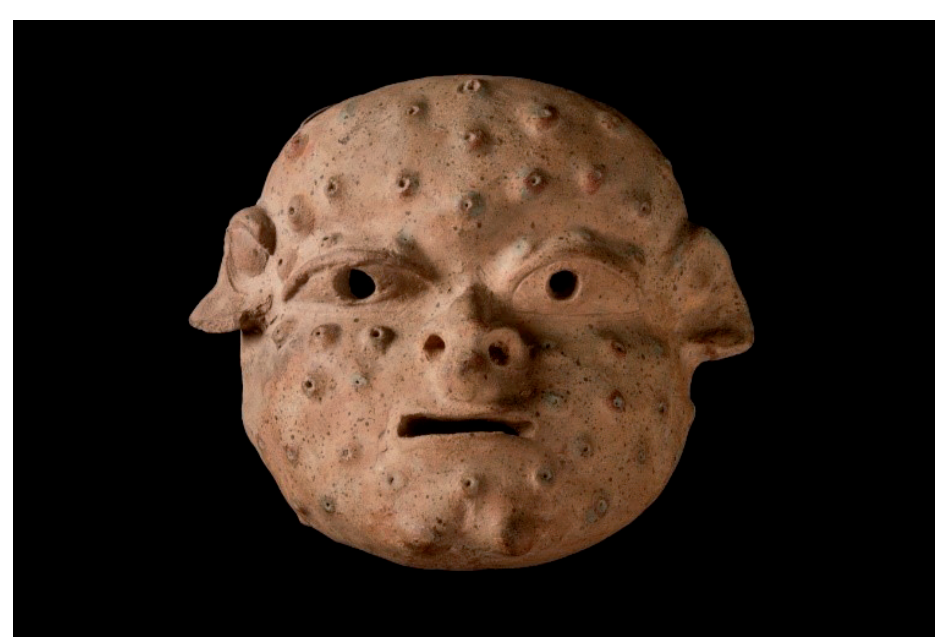

Figura 1. Máscara en cerámica de la cultura Jama-Coaque (400 a.C a 400 d.C) con lesiones nodulares por verruga peruana (bartonelosis).Colección del autor. Fotografía tomada por Andrea Mejía Cruz.
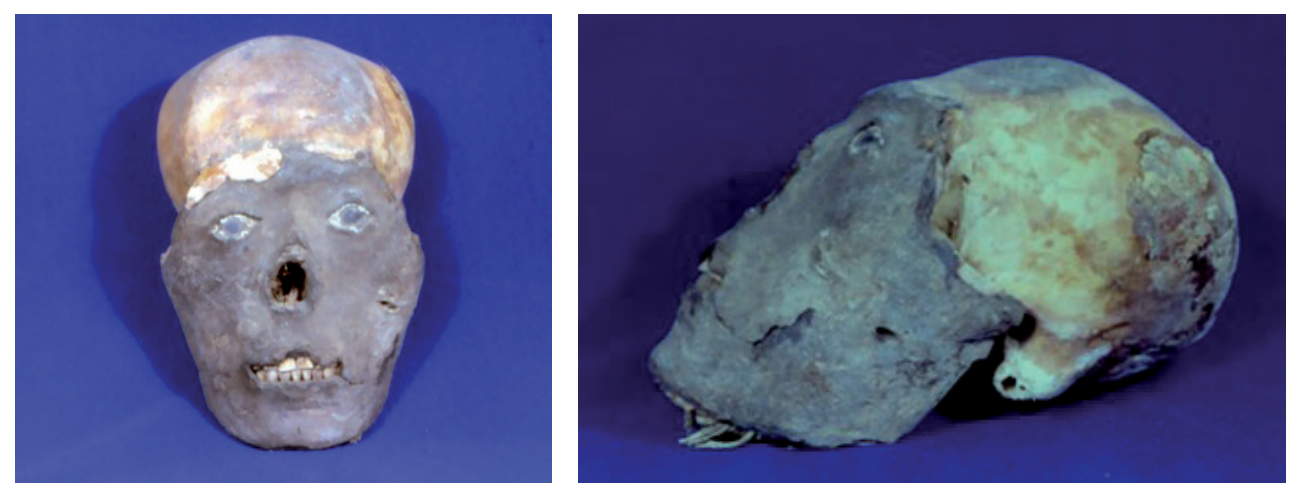

Figuras 2 y 3. Vistas frontal y lateral izquierda de calavera enmascarada Yupko-Yupka (motilones). Colección del ICANH. Fotografía tomada por el autor.

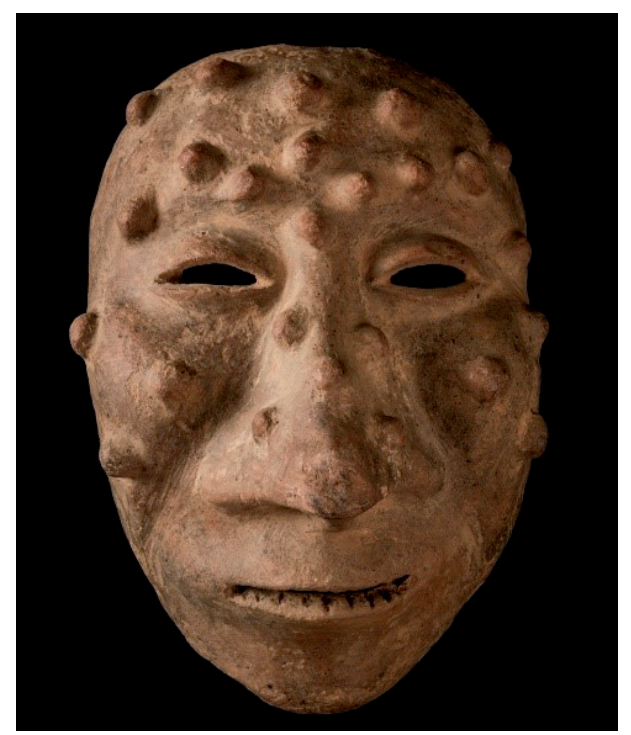

Figura 4. Máscara en cerámica perteneciente a la cultura Nariño, del Carchi o de los Pastos, probablemente del horizonte Capulí. (500.d.C -1.500 d.C), con un brote tipo nodular compatible con la bartonelosis en su fase verrucosa. Colección del autor. Fotografía tomada por Andrea Mejía Cruz.

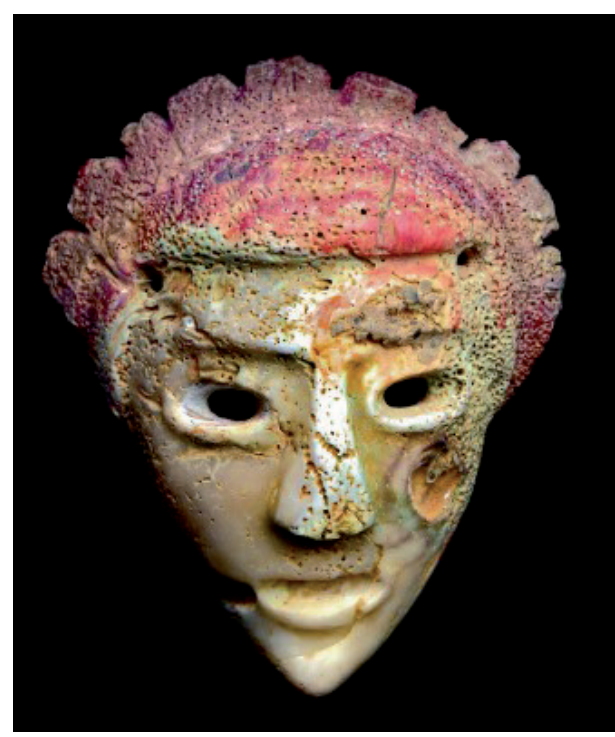

Figura 5. Máscara esculpida en concha Spondylus princeps. Fotografía cedida por cortesía de Alonso Restrepo de León, de su libro inédito "Los Rostros de la Mar". 


\section{MATERIALES Y MÉTODOS}

Se presentan cuatro máscaras, tres ecuatorianas y una colombiana. Las ecuatorianas de culturas que florecieron en la costa de ese país, son dos de cerámica y una de concha. La colombiana es de cobre y fue elaborada en el interior de la que se llama cultura Quimbaya. Las dos primeras máscaras, por informes de la persona que me facilitó las piezas fueron obtenidas en la región donde floreció la cultura precolombina ecuatoriana Jama-Coaque. La primera de ellas es de cerámica, de un tamaño de $21 \mathrm{~cm}$ de largo, por $20 \mathrm{~cm}$ de ancho, que al cubrir perfectamente el rostro de una persona, debió ser una máscara mortuoria por su peso y tamaño, tiene un agujero en cada extremo superior -posiblemente para sujetarla al rostro del difunto-, un asa en medio de la frente -posiblemente para levantar la máscara- y en reemplazo de los ojos sendas incrustaciones de piedras verdes, casi semicirculares, y otra incrustación de piedra de color verde, aunque más brillante, en región sublabial un bezote. Estas incrustaciones, muy probable de piedra jadeíta, contrastan vivamente con la simulación de dientes realizada con siete piedras o material malacológico que muestra en la mitad de las cinco centrales una canalura, tratando de señalar los incisivos inferiores y superiores, aunque anatómicamente son cuatro y cuatro, aquí se muestran cinco y cinco, y tiene sendos colmillos superiores bien proporcionados al tamaño de los incisivos. En el ángulo de la comisura labial izquierda se aprecia un fragmento del mismo material que simula los dientes. Todas estas características señalan que su destinario era un personaje de poder. La máscara muestra una línea de fractura que atraviesa toda su longitud próxima al lado derecho de la nariz y dos líneas de fracturas perpendiculares, la primera en la región por debajo del arco superciliar derecho y la segunda a la altura media de la zona mentoniana derecha. Las tres fracturas están arregladas con material de adhesión (figura 6).

La segunda máscara es de una cerámica color café, de 15 $\mathrm{cm}$. de largo por $14 \mathrm{~cm}$ de ancho, que muestra en la frente diez nódulos y en cada mejilla tres, para un total de dieciséis lesiones compatibles con los nódulos hemangiomatosos de la verruga peruana o bartonelosis (figura 7).

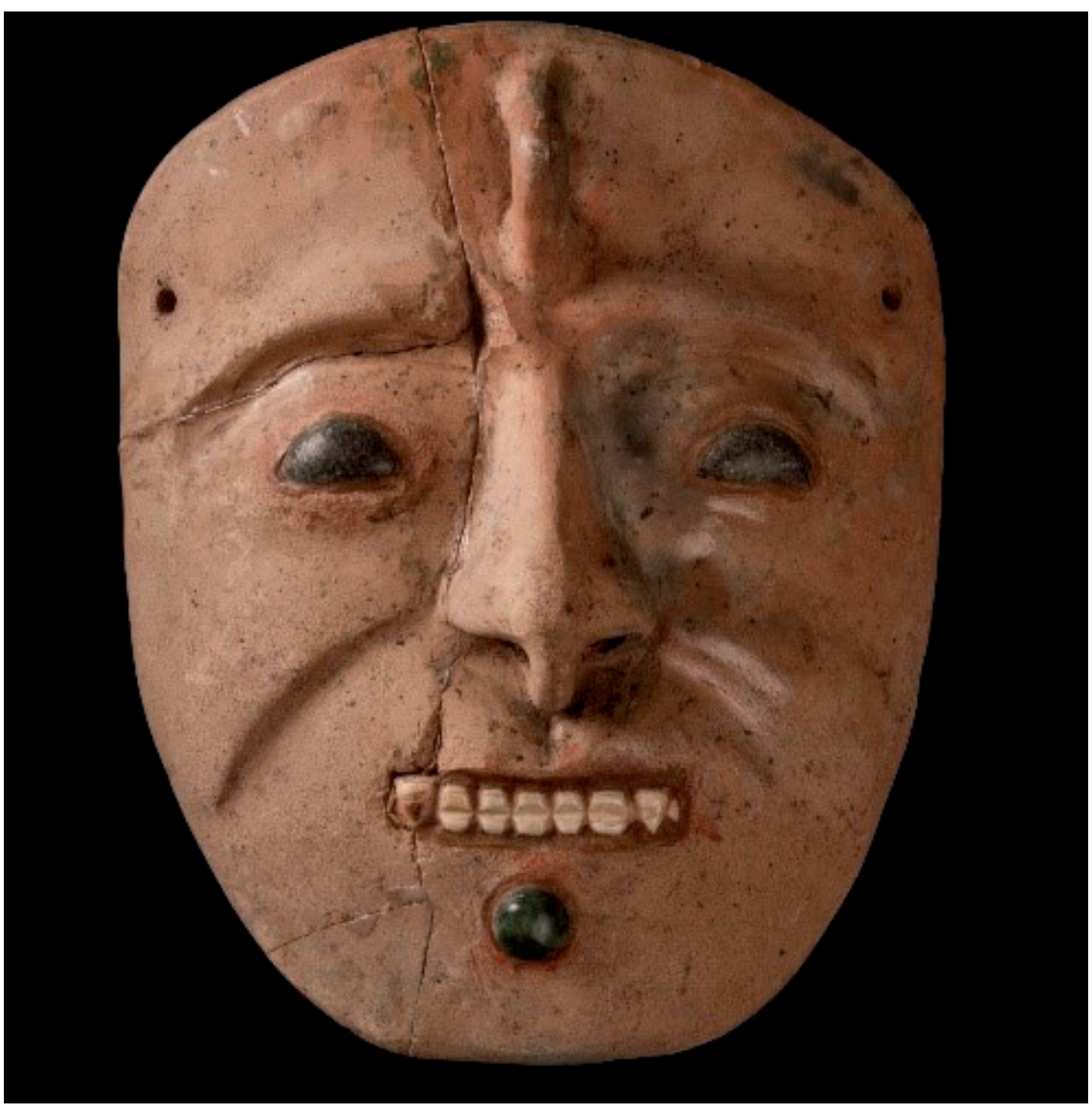

Figura 6. Máscara mortuoria Jama-Coaque (400 a.C - 400 d.C). Colección del autor. Fotografía tomada por Andrea Mejía Cruz. 


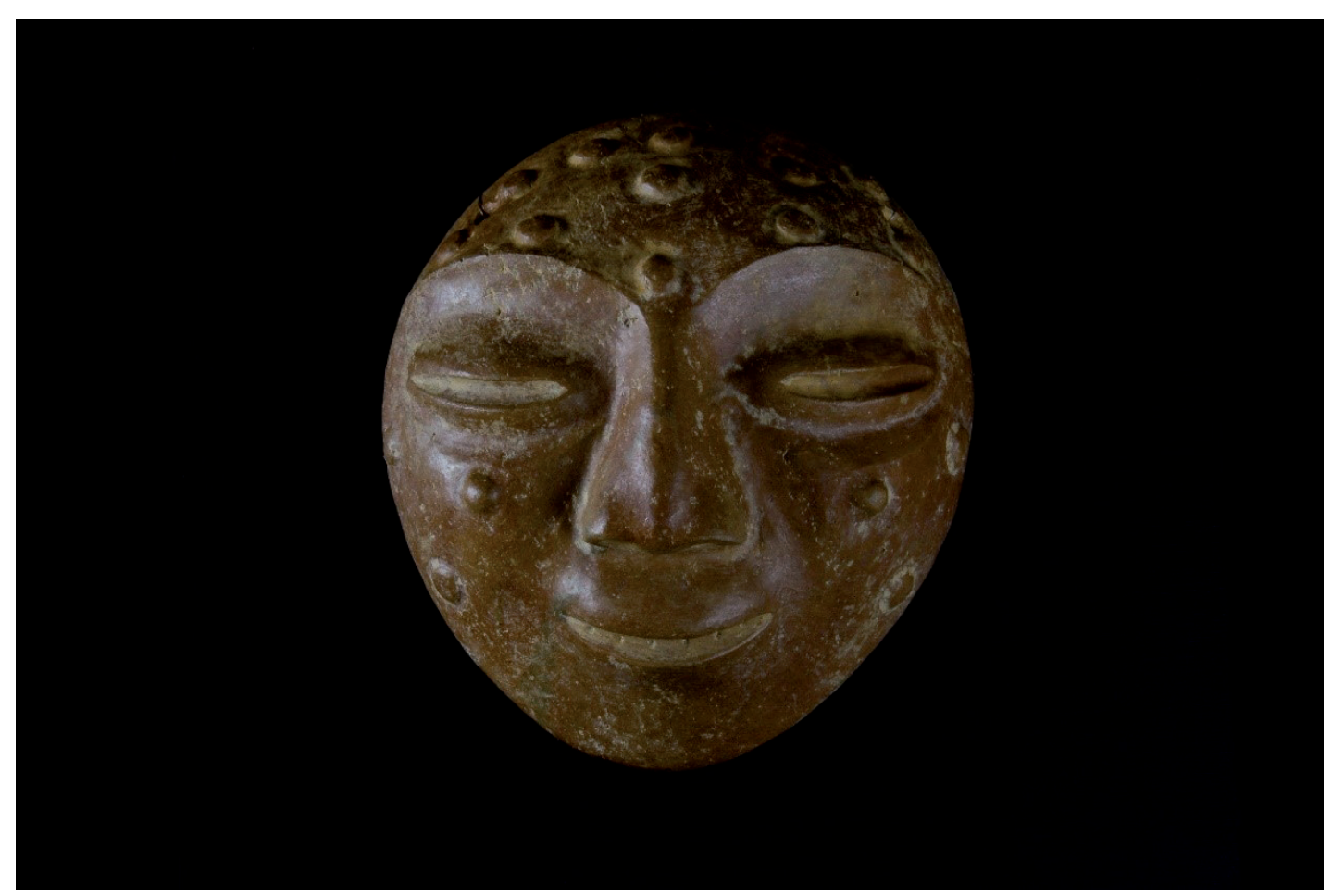

Figura 7. Máscara en cerámica de la cultura Jama-Coaque (400 a. C a 400 d.C) que muestra una erupción tipo nódulos en frente y mejillas, compatible con bartonelosis en su fase de verruga. Colección del autor. Fotografía tomada por Andrea Mejía Cruz.

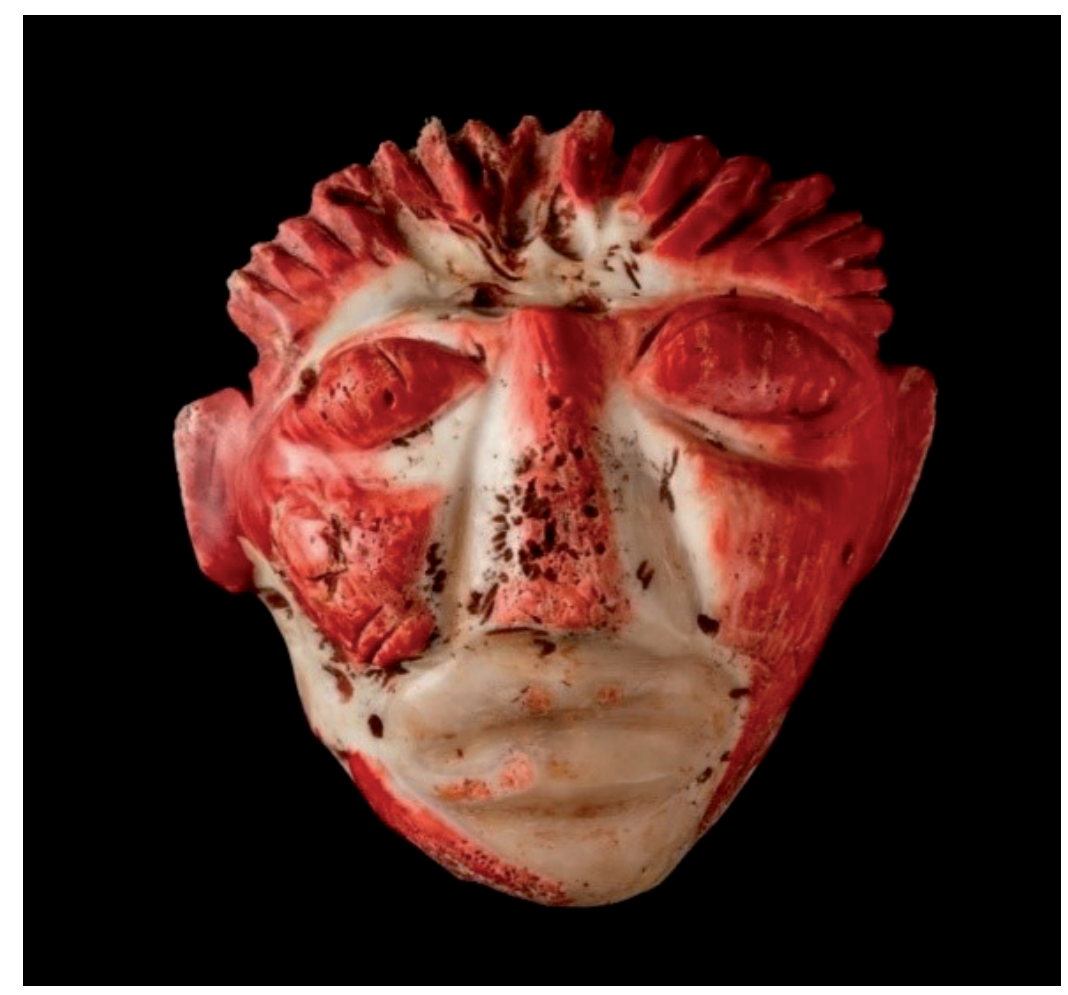

Figura 8. Máscara ecuatoriana esculpida en concha Spondylus prínceps de $15 \mathrm{~cm}$ de altura, 9 de anchura y $5 \mathrm{~cm}$ de profundidad, Colección del autor. Fotografía tomada por Andrea Mejía Cruz. 


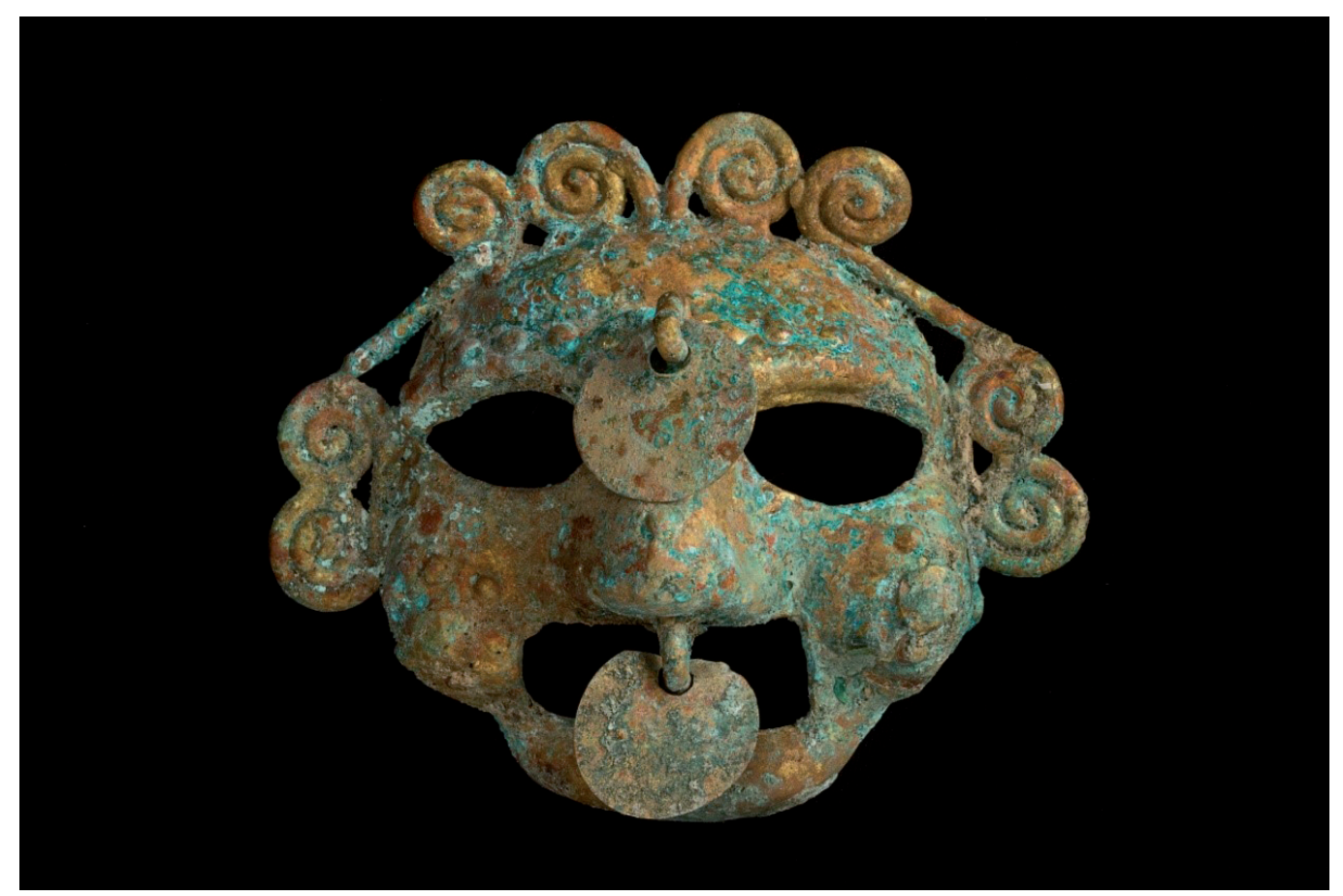

Figura 9. Máscara en cobre de la cultura Quimbaya (la fase tardía de 650 d.C a 1600 d.C) que muestra ocho volutas, cuatro en la cabeza y dos a la altura de las orejas, un adorno redondo que pende de la glabela y otro redondo del mismo tamaño que el anterior que hace de nariguera. El personaje tiene la boca abierta, presenta los orificios oculares libres y unos pómulos prominentes. Colección del autor. Fotografía tomada por Andrea Mejía Cruz.

La tercera máscara, también ecuatoriana está esculpida en la concha roja del Spondylus prínceps, fue elaborada por algunos de los pueblos o culturas prehispánicas del Ecuador. Su altura es de15 cm, su ancho es de $9 \mathrm{~cm}$ y su profundidad es de $5 \mathrm{~cm}$. Tiene dos colores el rojo y el blanco.

Con alta probabilidad fue una máscara propiciatoria o un exvoto, por ser elaborada en esa concha tan cargada de simbolismos (figura 8).

La cuarta máscara es de cobre, verde con amarillo, de la región del Cauca medio o territorio Quimbaya, parece ser del periodo tardío (650 d.C- a 600 d.C) mide $11 \mathrm{~cm}$ de ancho, $9 \mathrm{~cm}$ de alto y $5 \mathrm{~cm}$ de profundidad, muestra ocho volutas, cuatro en la cabeza y dos a la altura de las orejas, un adorno redondo que pende de la glabela y otro redondo del mismo tamaño que el anterior que hace de nariguera. El personaje tiene la boca abierta, presenta los orificios oculares libres y unos pómulos prominentes. Posiblemente fue llevada pendiendo del cuello si se acepta que los dos pequeños orificios que están entre las volutas 1 y 2; 3 y 4 fueron usados para pasar un cordón (figura 9)

\section{IS C USIÓN}

La denominada cultura Jama-Coaque ocupó en la costa pacífica de Ecuador el norte de la provincia de Manabí y el sur de la provincia de Esmeraldas, entre la bahía de
Caráquez al sur y el cabo de San Francisco, al norte, durante un largo periodo desde el 350 a.C al 1533 d.C. Esta ocupación tiene dos periodos, el Jama-Coaque (400 a.C al 400 d.C) que corresponde al periodo de Desarrollo Regional de la arqueología ecuatoriana, y Jama-Coaque II desde el 400 d.C hasta el temprano periodo colonial que corresponde a la Integración según la arqueología del Ecuador. ${ }^{6}$

Se sabe que las máscaras, así como las figuras con trajes emplumados, las hibridaciones antropozoomorfas, los animales con lengua bífida y las figurillas articuladas pertenecieron a la sociedad Jama-Coaque I y que esta tuvo contactos comerciales con las sociedades mesoamericanas. ${ }^{7}$

Aunque se sabe que las culturas de la costa Pacífica del Ecuador desde la de Valdivia, las del complejo formativo Chorrera-Machalilla y las de los desarrollos regionales como Guangala, Jama-Coaque, La Tolita, Bahía, Manta y Huancavilca, tuvieron una especial relación con la concha Spondylus prínceps por su estrecha asociación con las temperaturas de la corriente de Humboldt y su valor predictivo de los regímenes de invierno o verano y de los fenómenos de El Niño y La Niña, poco se sabe de la tipología artística de esas culturas al trabajar esa concha, a excepción de la sencillez de las producidas por la de Valdivia. La razón anterior impide asociar la máscara de concha aquí presentada con cualquiera de las culturas señaladas? ${ }^{7}$ Aquí vale la pena contar algo de lo que Alonso Restrepo de León (q.e.p.d), el amigo y gran coleccionista y 


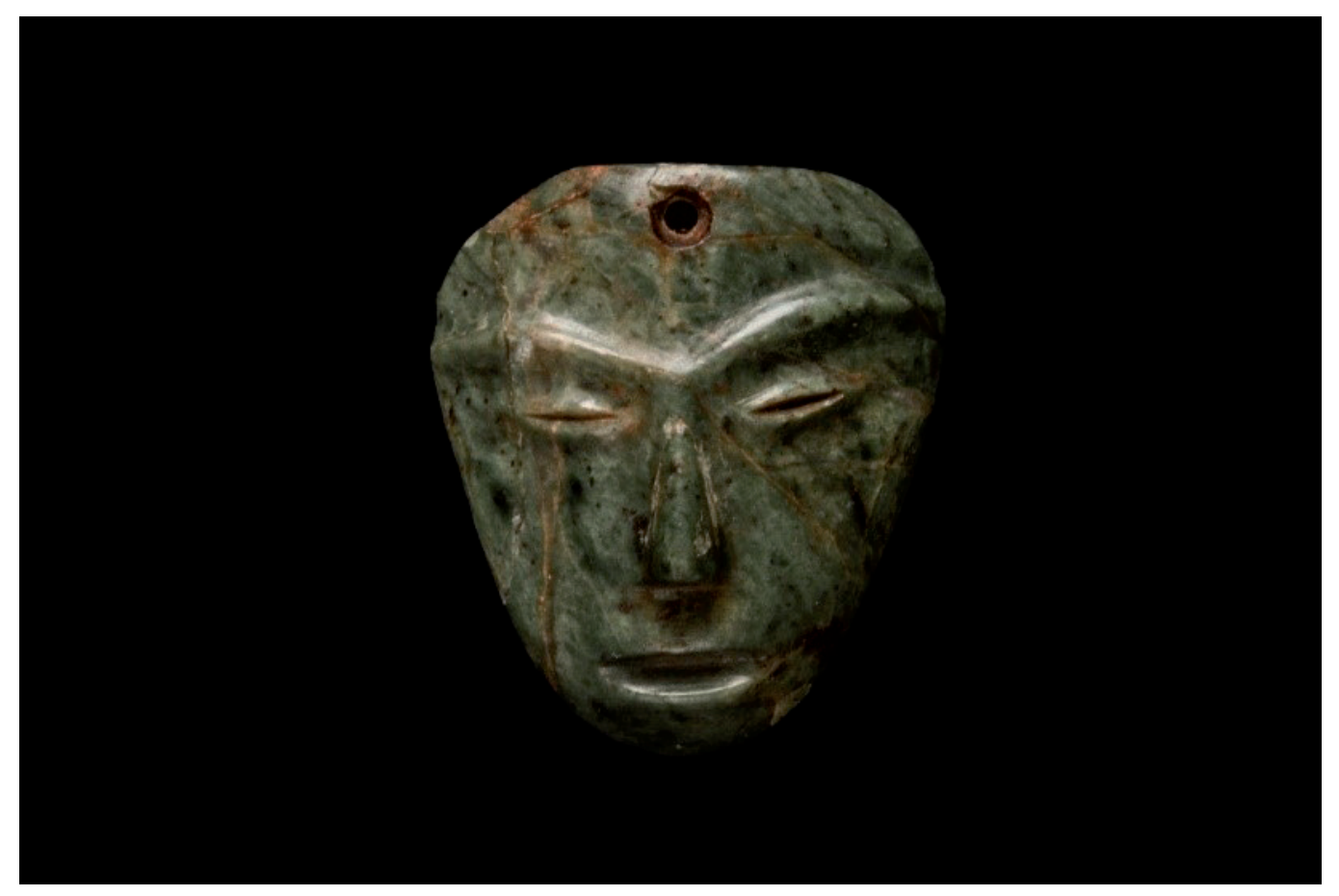

Figura 10. Máscara Nariño de jade. Colección del autor. Fotografía tomada por Andrea Mejía Cruz.

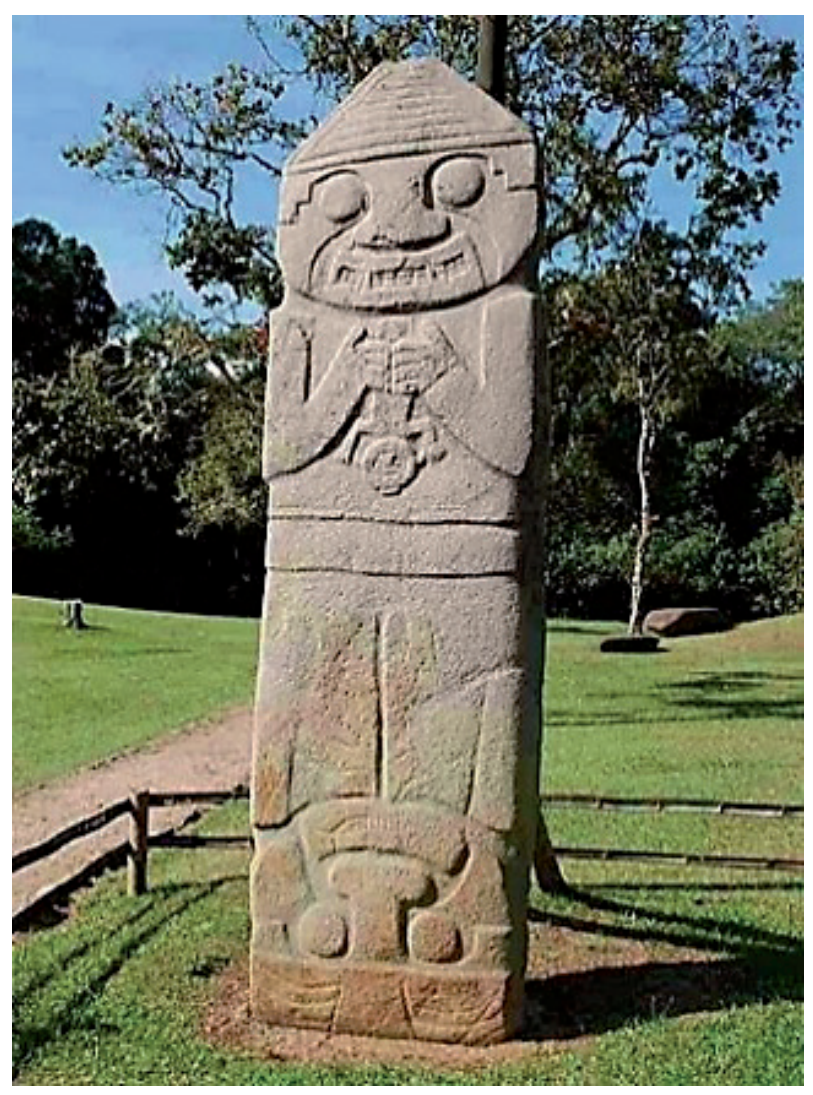

Figura 11. Escultura de San Agustín que muestra a un personaje adulto que toma por las piernas a un niño pequeña en un ritual de un sacrificio infantil. Fotografía tomada por el autor. 
estudioso de la malacología y yo tuvimos oportunidad de saber sobre esta concha de tan importante valor. En vista de que no encontrábamos suficiente información sobre las máscaras elaboradas en esa concha, Alonso, poseedor de más de trescientas de esas máscaras, logró que la arqueóloga ecuatoriana y encargada de una sección de las oficinas de patrimonio del Ecuador, Estelina Quinatoa viajara a Bogotá a conocer la colección. Estelina al ver la colección quedó perpleja y nos contó que en su país en los museos no había más de veinte de esas máscaras y que no existía un estudio sistemático sobre ellas ${ }^{7}$.

De la máscara colombiana, del Cauca medio, en donde se asentó lo que se ha llamado estilo Quimbaya, se sabe que en ese estilo hubo una predilección en el periodo tardío, 650 a 1600 d.C, por los adornos corporales en cobre, así como hubo una predilección por figuras humanas, fitomorfas y de recipientes en oro en el periodo temprano, 550 a.C a 650 d.C. ${ }^{8}$ Con seguridad fue considerada de gran valor y por esto mismo enterrada como parte de un ajuar funerario. Las diferentes sociedades han considerado el cadáver ya sea como el último portador del alma que emigra, una fuente de impureza o como una oportunidad de resaltar el poder del difunto o de seguir castigándolo y solo en la cultura occidental para enseñar anatomía en la escuela de Alejandría, en el periodo helenístico, y luego en Europa. Todas las sociedades han tenido ritos fúnebres como por ejemplo los vinculados con las posiciones del cadáver en el entierro, cremación, inhumación y entierros secundarios. Los mausoleos, las pirámides, los procesos de momificación, los días de duelo, los convites a comer durante la celebración del duelo eran trabajos asociados para resaltar el poder del difunto, como lo fueron los acompañantes de los muertos como una de las esposas, algunos sirvientes, animales domésticos como el gato en el Antiguo Egipto, el caballo entre los habitantes de las grandes estepas asiáticas, y los perros en México y Perú precolombinos, y el entierro junto al cadáver de elementos como armas, joyas y máscaras.

Las máscaras grandes y pesadas, en especial las de piedras, fueron usadas para resaltar el poder del difunto y las de menor peso usadas en vida como adorno por los personajes de alto estatus y poder en las sociedades y luego cuando se consideraban de gran valor enterrarlas junto al cadáver. Las piedras verdes como el jade y la jadeíta, tan usadas por los indígenas de mesoamérica, han sido asociadas con la fertilidad y el renacer. La pequeña máscara de jade perteneció a la cultura Nariño, en donde por tener un agujero en la frente es evidente que se usó como un adorno colgante de gran valor sobre el pecho para resaltar el estatus de quien lo lucía (figura 10).

El cobre fue un material empleado en diferentes momentos y sociedades en América. En Colombia se utilizó en aleaciones con oro- tumbaga- o solo, en la cultura Zenú se hicieron cascabeles y en la cultura Nariño, discos y flautas de pan o rondadores.

A las partes del cuerpo las diferentes sociedades les han dado un significado similar o diferente. Por ejemplo la cabellera abundante se ha asociado con unas extensas raíces capaces de permitir el crecimiento de la plantas, de allí la asociación de la cabellera con la fertilidad y la sexualidad y por eso el afán de cubrir la cabellera de la monjas y de las mujeres islámicas; las gibas o jorobas se han asociado con la acumulación, el intestino grueso con la retención y la avaricia, el bazo con la melancolía, el hígado con la ira, la espalda con la rectitud y la dentadura bien alineada con el orden y la templanza, los colmillos con la fuerza y la agresión, como es el caso de las bocas y dentaduras de la estatuaria de San Agustín (figura 11).

\section{CONCLUSIONES}

Se presentan cuatro máscaras nuevas, dos de cerámica, muy probable Jama-Coaque, la primera posiblemente mortuoria puede cubrir el rostro de un adulto, que por sus incrustaciones de piedras verdes en el lugar de los ojos y en la región sublabial y la simulación de unos dientes alineados y con colmillos de tamaño normal representa a un sujeto con poder espiritual y de mando y la segunda, que podría ocultar el rostro de un adulto más pequeño afectado por la fase verrucosa de la bartonelosis. Una tercera de concha también ecuatoriana, talvez sea de carácter votivo y una cuarta colombiana de cobre del Cauca medio Quimbaya, usada como adorno corporal.

De las tres máscaras una proviene de las montañas del sur de Colombia y muestra la bartonelosis en su fase verrucosa o nodular, producida por Bartonella bacilliformis transmitida por la picadura de la hembra del mosquito phlebotomo (Lutzomyia spp), demuestran cómo esta enfermedad se presenta en diferentes altitudes o pisos térmicos propios de los valles interandinos como los descritos en Oroya, Perú y Guáitara, Colombia, así como en la costa pacífica, sitio donde se ubican las localidades ecuatorianas Jama y Coaque. Esta enfermedad está restringida a Colombia, Ecuador y Perú.

Pedro Cieza de León describió en la costa ecuatoriana "se crían en los hombres unas verrugas bermejas del grandor de nueces, y les nascen en la frente y en las narices y en otras partes; que, además de ser mal grave, es mayor la fealdad que hacen en los rostros, y créese que de comer algún pescado procede este mal". ${ }^{9}$

Otra descripción de esta enfermedad la hizo el Inca Garcilaso en su Historia General del Perú, tomo 1, cap. XV. En 1531 las fuerzas expedicionarias de los españoles hicieron un alto en la bahía de Coaqui (Coaque-Ecuador): "... se les recreció a los de Pizarro una enfermedad extraña y abominable, y fue que les nacían por la cabeza, por el rostro y por todo el cuerpo unas como verrugas que lo parecían al principio cuando se les mostraban; más después, yendo creciendo, se ponían como brevas prietas y del tamaño dependían de un pezón, destilando de si mucha sangre, causando grandísimo dolor y horror, no se dejaban tocar, ponían feísimos a los que daban, porque unas verrugas 
colgaban de la nariz, de las barbas y orejas, no sabían qué les hacer; murieron muchos, otros muchos sanaron; no fue la enfermedad general por todos los españoles, aunque corrió por todo el Perú, que muchos años después vi en el Cuzco tres o cuatro españoles con la misma enfermedad y sanaron.." ${ }^{10}$ El cronista Miguel de Estete afirmó que "el pueblo de Coaque es la costa más enferma que hay debajo de cielo". ${ }^{11}$

La bartonelosis conforma con la tungiasis, la enfermedad de Chagas, las rickettsiosis por Rickettsia rikettssi transmitidas por garrapatas (fiebre de las Montañas Rocosas, fiebre de Tobia, fiebre de Sao Paulo) y el carate o pinta o mal del pinto o jovero o purupuru, las cinco enfermedades propias o autóctonas de América prehispánica. En América además de la treponematosis autóctona del carate, existieron las otras tres treponematosis: la sífilis venérea, el pian o yaws o frambesía y se conoció la tuberculosis hominis, no así la bovis. La paleopatología tiene tres fuentes principales para su estudio: los restos humanos (huesos, momias, coprolitos, etc), el arte y la historia. La presentación aquí mostrada tiene dos de las tres fuentes, el arte y la historia. La paleopatología es uno de los cimientos de la paleoepidemiología, como a su vez ésta conforma un capítulo más de la epidemiologia general con la epidemiología histórica, la etnoepidemiología y la epidemiología intercultural.

El realismo en las representaciones artísticas de la bartonelosis aquí mostrada, al igual que el que se utilizó en otras patologías, a decir de la medicina moderna, buscaba mostrarle a los miembros de la comunidades indígenas con seguridad las diferencias en la anatomía de ellas, dentro una medicina empírico analógica a manera como la medicina occidental lo hace con las colecciones de teratología, los moldes en cera de las enfermedades y la fotografía, dentro de una medicina puramente empírica y analítica. ${ }^{12-24}$

En toda América se hicieron ofrendas votivas y el adorno personal fue utilizado para establecer jerarquías de poder y estatus.

\section{REFERENCIAS}

1. Sotomayor Tribín HA. A propósito de la Bartonelosis en una Máscara Ecuatoriana Precolombina. Revista Medicina. 1994;16(2):28-9.

2. Sotomayor Tribín HA, Correal Urrego G. Las calaveras enmascaradas de las momias Yuko-Yukpa (Motilones). Revista de la Academia Colombiana de Ciencias Exactas, Fisicas y Naturales. 2003;27(102):5-14.

3. Sotomayor Tribín HA. Aspectos históricos y geográficos de algunas enfermedades importantes en Colombia. Bogotá: Universidad Militar Nueva Granada; 2012.

4. Chávez Mendoza Á. Máscara precolombina. Bogotá: Ediciones Zazacuabi; 1977.

5. Restrepo De León A. Los rostros de la mar. Bogotá: Inédito; 2017.

6. Quinatoa Cotacachi E. Representaciones ancestrales y colores del cosmos: diseños de platos del Carchi. Colombia: Ministerio de Cultura y Patrimonio; 2013.

7. Museo Nacional del Banco Central del Ecuador. Jama-Coaque en la Guía de la sala de arqueología. Quito: Banco Central del Ecuador; 2001. p. 62.

8. Botero CI. Museo del Oro. Bogotá: Banco de la República; 2008. p. 91-103.

9. de León C. La crónica del Perú. Madrid: Historia 16; 1984.

10. Garcilaso de la Vega I. Historia General del Perú. In: Oviedo JM, editor. La edad del oro Crónicas y testimonios de la conquista del Perú. Barcelona: Tusquet; 1986. p. 205.

11. Flores E, Cevallos L, Burstein Z. Historia de la dermatología en Perú. In: Galimberti R, Pierini A, Cervini A, editors. Historia de la Dermatología Latinoamericana. Toulouse: Ed Privat; 2007. p. 307-64. 